Bull. Austral. Math. Soc.

$46 \mathrm{H} 05,46 \mathrm{H} 10,47 \mathrm{~A} 10,06 \mathrm{~F} 25$

VOL. $74(2006) \quad$ [239-246]

\title{
ON THE BOUNDARY SPECTRUM IN BANACH ALGEBRAS
}

\author{
S. Mouton
}

\begin{abstract}
We investigate some properties of the set $S_{\partial}(a)=\{\lambda \in \mathbb{C}: \lambda-a \in \partial S\}$ (which we call the boundary spectrum of $a$ ) where $\partial S$ denotes the topological boundary of the set $S$ of all non-invertible elements of a Banach algebra $A$, and where $a$ is an element of $A$.
\end{abstract}

\section{INTRODUCTION AND PRELIMINARIES}

Let $A$ be a complex Banach algebra with unit 1. We shall denote the spectrum of an element $a$ in $A$ by $\sigma(a)$ and the spectral radius of $a$ in $A$ by $r(a)$ (or by $\sigma(a, A)$ and $r(a, A)$ respectively, if the particular Banach algebra needs to be emphasized). The distance from an element $\alpha \in \mathbb{C}$ to a subset $E$ of $\mathbb{C}$ will be denoted by $d(\alpha, E)$, and $\delta(a)$ (or $\delta(a, A)$, if necessary) will indicate the distance $d(0, \sigma(a))$ from 0 to the spectrum of $a$. If $\lambda \in \mathbb{C}$, then we shall write $\lambda$ for the element $\lambda 1$ in $A$. We recall that if $\alpha \notin \sigma(a)$, then $d(\alpha, \sigma(a))=1 /\left(r\left((\alpha-a)^{-1}\right)\right)([\mathbf{1}$, Theorem 3.3.5]).

If $E$ is a subset of a metric space $\mathcal{X}$, then $\partial_{\mathcal{X}} E$ denotes the topological boundary of $E$ and int $_{\mathcal{X}} E$ the topological interior of $E$ relative to $\mathcal{X}$. For an $r>0$ and an element $x$ in $\mathcal{X}$, the notation $B_{\mathcal{X}}(x, \varepsilon)$ will be used to denote the open ball relative to $\mathcal{X}$ with centre $x$ and radius $\varepsilon$. (If the choice of a metric space $\mathcal{X}$ is clear, the subscript $\mathcal{X}$ will be dropped.)

In this paper we consider the set $S_{\partial}(a)=\{\lambda \in \mathbb{C}: \lambda-a \in \partial S\}$ (or $S_{\partial}(a, A)$, if the particular Banach algebra needs to be emphasized) where $S$ (or $S_{A}$, if necessary) denotes the set of all non-invertible elements of $A$. Some properties of this set are investigated: in particular the relationship between $S_{\partial}(a, A)$ and $S_{\partial}(a, B)$ where $B$ is a closed subalgebra of a Banach algebra $A$ such that $B$ contains the unit of $A$, and the relationship between $S_{\partial}(a, A)$ and $S_{\partial}(T a, B)$ where $B$ is another Banach algebra and $T: A \rightarrow B$ a homomorphism. Finally, some results involving the boundary spectrum $S_{\partial}(a)$ of a positive element $a$ in an ordered Banach algebra are obtained.

Received 4th April, 2006

Copyright Clearance Centre, Inc. Serial-fee code: 0004-9727/06 \$A2.00+0.00. 


\section{BOUNDARY SPECTRUM}

Let $A$ be a complex Banach algebra with unit 1 and let $S$ be the set of all noninvertible elements of $A$. Then $S$ is a closed subset of $A$. Define, for $a \in A$, the set $S_{\partial}(a)$ in the complex plane as follows:

$$
S_{\partial}(a)=\{\lambda \in \mathbb{C}: \lambda-a \in \partial S\}
$$

We shall call this set the boundary spectrum of $a$ in $A$. Also define, for $a \in A$,

$$
r_{1}(a)=\sup \{|\lambda|: \lambda \in \partial \sigma(a)\}
$$

and

$$
r_{2}(a)=\sup \left\{|\lambda|: \lambda \in S_{\partial}(a)\right\}
$$

Proposition 2.1. Let $A$ be a Banach algebra and $a \in A$. Then $\partial \sigma(a)$ $\subseteq S_{\partial}(a) \subseteq \sigma(a)$; and therefore $r_{1}(a)=r_{2}(a)=r(a)$, and if $\alpha \notin \sigma(a)$, then $d(\alpha, \partial \sigma(a))$ $=d\left(\alpha, S_{\partial}(a)\right)=d(\alpha, \sigma(a))$.

PROOF: To prove that $\partial \sigma(a) \subseteq S_{\partial}(a)$, let $\lambda \in \partial \sigma(a)$ and $\varepsilon>0$. Then there exist a $\lambda_{1} \in B(\lambda, \varepsilon) \cap \sigma(a)$ and $a \lambda_{2} \in B(\lambda, \varepsilon) \cap(\mathbb{C} \backslash \sigma(a))$. If $b_{1}=\lambda_{1}-a$ and $b_{2}=\lambda_{2}-a$, then $b_{1} \in S, b_{2} \notin S$ and $b_{1}, b_{2} \in B(\lambda-a, \varepsilon)$. Therefore $\lambda-a \in \partial S$, so that $\lambda \in S_{\partial}(a)$. This proves that $\partial \sigma(a) \subseteq S_{\partial}(a)$, and since $S$ is closed, $\partial S \subseteq S$, so that $S_{\partial}(a) \subseteq \sigma(a)$.

It follows from Proposition 2.1 that, for every $a \in A$, the set $S_{\partial}(a)$ is non-empty. Since $\partial S$ is closed, $S_{\partial}(a)$ is closed, and since $S_{\partial}(a)$ is contained in the spectrum of $a$, it is bounded as well; in fact, $S_{\partial}(a) \subseteq\{\lambda \in \mathbb{C}:|\lambda| \leqslant r(a)\}$. Therefore $S_{\partial}(a)$ is a compact set.

In general, $\partial \sigma(a) \neq S_{\partial}(a)$. We proceed to illustrate this with an example.

EXAMPLE 2.2. ([1, Remark 1, p.56]) Let $l^{2}(\mathbb{Z})$ be the Hilbert space of all bilateral square-summable sequences and $\left\{e_{n}: n \in \mathbb{Z}\right\}$ the orthonormal basis where, for each integer $n$, the vector $e_{n}$ is $\left(\ldots, \xi_{-1},\left(\xi_{0}\right), \xi_{1}, \ldots\right)$, where $\xi_{n}=1$ and $\xi_{i}=0$ for all integers $i$ different from $n$. (In this case, the term in round brackets indicates the one corresponding to index zero.) Let $T, R: l^{2}(\mathbb{Z}) \rightarrow l^{2}(\mathbb{Z})$ be the weighted shifts

$$
T e_{n}= \begin{cases}0 & \text { if } n=-1 \\ e_{n+1} & \text { if } n \neq-1\end{cases}
$$

and

$$
R e_{n}= \begin{cases}e_{0} & \text { if } n=-1 \\ 0 & \text { if } n \neq-1\end{cases}
$$

Then $0 \in \sigma(T)$ and $\sigma(T+\lambda R)$ is contained in the unit circle for all $\lambda \neq 0$. 
Moreover, if $0<|\lambda|<1$, then $\lambda$ is an eigenvalue of $T$. Indeed, if $\xi_{j}=0$ for all $j \in \mathbb{N} \cup\{0\}$ and $\xi_{-j}=\lambda^{j-1}$ for all $j \in \mathbb{N}$, then the (non-zero) element $\left(\ldots, \xi_{-1},\left(\xi_{0}\right), \xi_{1}, \ldots\right)$ of $l^{2}(\mathbb{Z})$ is in the kernel of $T-\lambda I$.

(The above facts about the operators $T$ and $R$ also follow from ([3, Problem 84]).)

EXAMPLE 2.3. Let $l^{2}(\mathbb{Z})$ be the Hilbert space of all bilateral square-summable sequences, $A$ the Banach algebra $\mathcal{L}\left(l^{2}(\mathbb{Z})\right)$ of all bounded linear operators on $l^{2}(\mathbb{Z})$ and $T$ the element of $A$ defined in Example 2.2. Then $\partial \sigma(T)$ is properly contained in $S_{\partial}(T)$.

Proof: Let $\left(\lambda_{n}\right)$ be a sequence different from zero converging to zero, $R$ the operator defined in Example 2.2 and $T_{n}=T+\lambda_{n} R(n \in \mathbb{N})$. Then $\left\|T_{n}-T\right\| \rightarrow 0$. Moreover, by Example 2.2, each $T_{n}$ is invertible and $T$ is not invertible. Hence $T \in \partial S$. Therefore $-T \in \partial S$, and so $0 \in S_{\partial}(T)$. However, Example 2.2 together with the remark thereafter imply that 0 is an interior point of $\sigma(T)$, and so $0 \notin \partial \sigma(T)$.

We recall the following well-known property of boundary points of the set of invertible (or non-invertible) elements:

ThEOREM 2.4. ([10, Theorem 2.5, p. 397]) Let $A$ be a Banach algebra and $a \in A$. If $a \in \partial S$, then $a$ is a topological divisor of zero.

From the above theorem we immediately obtain the following property of the boundary spectrum of $a$ :

COROLlary 2.5. Let $A$ be a Banach algebra and $a \in A$. If $\lambda \in S_{\theta}(a)$, then $\lambda-a$ is a topological divisor of zero.

Lemma 2.6. Let $A$ be a Banach algebra, $a \in \partial S$ and $d$ an invertible element. Then $a d \in \partial S$ and $d a \in \partial S$.

Proof: If $a \in \partial S$ and $d$ is invertible, then for each $\varepsilon>0$ there exist elements $c_{1} \in S \cap B(a,(\varepsilon /\|d\|))$ and $c_{2} \in(A \backslash S) \cap B(a,(\varepsilon /\|d\|))$. It follows that $c_{1} d \in S \cap B(a d, \varepsilon)$ and $c_{2} d \in(A \backslash S) \cap B(a d, \varepsilon)$. Hence $a d \in \partial S$, and similarly $d a \in \partial S$.

It follows from Lemma 2.6 that $a \in \partial S$ if and only if $\lambda a \in \partial S$, for all $\lambda \neq 0$.

Proposition 2.7. Let $a$ be an invertible element of a Banach algebra $A$. Then $S_{\partial}\left(a^{-1}\right)=\left(S_{\partial}(a)\right)^{-1}$.

Proof: For any $\lambda \neq 0$ and any invertible element $a \in A$ we have $\lambda-a^{-1}$ $=\lambda(a-(1 / \lambda)) a^{-1}$. So if $\lambda \in S_{\partial}\left(a^{-1}\right)$, then $\lambda(a-(1 / \lambda)) a^{-1} \in \partial S$. It follows from Lemma 2.6 that $a-(1 / \lambda) \in \partial S$, so that $1 / \lambda \in S_{\partial}(a)$. We have proved that $S_{\partial}\left(a^{-1}\right) \subseteq\left(S_{\partial}(a)\right)^{-1}$ for all invertible elements $a$, and therefore also $\left(S_{\partial}(a)\right)^{-1} \subseteq S_{\partial}\left(a^{-1}\right)$ for all invertible a.

Further mapping properties of $S_{\partial}$ will be investigated in a future paper.

Let $B$ be a closed subalgebra of a Banach algebra $A$ such that $B$ contains the unit element 1 of $A$. It is well known that if $a \in B$, then $\partial \sigma(a, B) \subseteq \partial \sigma(a, A)$ ([1, Theorem 
3.2.13]). We shall show that $S_{\theta}(a, B) \subseteq S_{\partial}(a, A)$ holds as well. In order to do this, we need the following results, some of which are interesting in their own right.

TheOREM 2.8. ([1, Theorem 3.2.13 (i)]) Let $B$ be a closed subalgebra of a Banach algebra $A$ such that $B$ contains the unit element 1 of $A$. Then $B \backslash S_{B}$ is the union of all components of $B \cap\left(A \backslash S_{A}\right)$ containing points of $B \backslash S_{B}$.

LEMMA 2.9. Let $B$ be a closed subalgebra of a Banach algebra $A$ such that $B$ contains the unit element 1 of $A$. If $E$ is a subset of $A$, then $\partial_{B} E \subseteq \partial_{A} E$.

THEOREM 2.10. Let $B$ be a closed subalgebra of a Banach algebra $A$ such that $B$ contains the unit element 1 of $A$. Then $S_{B}$ is the union of $S_{A}$ and all the components of $B \cap\left(A \backslash S_{A}\right)$ containing points of $S_{B}$.

Proof: Clearly $S_{A} \subseteq S_{B}$. If $x \in S_{B}$ and $x \notin S_{A}$, then $x \in B \cap\left(A \backslash S_{A}\right)$, so that $x$ is contained in a component of $B \cap\left(A \backslash S_{A}\right)$ which contains points of $S_{B}$. Hence $S_{B}$ is contained in the union of $S_{A}$ and all the components of $B \cap\left(A \backslash S_{A}\right)$ containing points of $S_{B}$.

Conversely, let $\Omega$ be a component of $B \cap\left(A \backslash S_{A}\right)$ which contains points of $S_{B}$. If $\Omega \nsubseteq S_{B}$, then $\Omega$ is a component of $B \cap\left(A \backslash S_{A}\right)$ which contains a point of $B \backslash S_{B}$. Theorem 2.8 implies that $\Omega \subseteq B \backslash S_{B}$, which contradicts the fact that $\Omega$ contains points of $S_{B}$. Hence $\Omega \subseteq S_{B}$.

The following result was proved in [2], using the fact that boundary points of the set of invertible elements of a Banach algebra are topological divisors of zero (see Theorem 2.4) and therefore permanently singular. We provide an alternative proof.

TheOREM 2.11. ([2, Corollary 18, p. 14]) Let $B$ be a closed subalgebra of a Banach algebra $A$ such that $B$ contains the unit element 1 of $A$. Then $\partial_{B} S_{B} \subseteq \partial_{A} S_{A}$.

Proof: To prove that $\partial_{B} S_{B} \subseteq \partial_{B} S_{A}$, suppose that $x \notin \partial_{B} S_{A}$. If $x \notin B$, then $x \notin \partial_{B} S_{B}$, so suppose that $x \in B$. Then there exists an $\varepsilon>0$ such that either (i) $B_{B}(x, \varepsilon) \subseteq S_{A}$ or (ii) $B_{B}(x, \varepsilon) \subseteq B \backslash S_{A}$. Since $S_{A} \subseteq S_{B}$, case (i) implies that $B_{B}(x, \varepsilon)$ $\subseteq S_{B}$, so that $x \notin \partial_{B} S_{B}$, so suppose that $B_{B}(x, \varepsilon)$ is contained in a component $\Omega$ of $B \cap\left(A \backslash S_{A}\right)$. If $\Omega$ contains points of $S_{B}$, then by Theorem $2.10, \Omega$ is contained in $S_{B}$, so that $x \notin \partial_{B} S_{B}$. If $\Omega$ contains no points of $S_{B}$, then $\Omega \subseteq B \backslash S_{B}$, so that once again, $x \notin \partial_{B} S_{B}$.

We have proved that $\partial_{B} S_{B} \subseteq \partial_{B} S_{A}$. Together with Lemma 2.9 the result follows. $\square$

Corollary 2.12. Let $B$ be a closed subalgebra of a Banach algebra $A$ such that $B$ contains the unit element 1 of $A$. If $a \in B$, then $S_{\partial}(a, B) \subseteq S_{\partial}(a, A)$.

Proof: If $\lambda \in S_{\partial}(a, B)$, then $\lambda-a \in \partial_{B} S_{B}$. It follows from Theorem 2.11 that $\lambda-a \in \partial_{A} S_{A}$, so that $\lambda \in S_{\partial}(a, A)$.

Now we consider the situation where $A$ and $B$ are Banach algebras (with $B$ not necessarily a subalgebra of $A$ ) and $T: A \rightarrow B$ a homomorphism, and investigate the 
relationship between $S_{\partial}(a, A)$ and $S_{\partial}(T a, B)$, where $a \in A$. We first establish some properties involving $T S_{A}$ and $S_{B}$, and $T\left(\partial_{A} S_{A}\right)$ and $\partial_{B} S_{B}$. The proof of the next lemma is trivial:

Lemma 2.13. Let $A$ and $B$ be Banach algebras and $T: A \rightarrow B$ a homomorphism. Then the following hold:

1. $T^{-1} S_{B} \subseteq S_{A}$.

2. If $T$ is surjective, then $S_{B} \subseteq T S_{A}$.

3. If $T$ is bijective, then $T^{-1} S_{B}=S_{A}$ and $T S_{A}=S_{B}$.

Theorem 2.14. Let $A$ and $B$ be Banach algebras and $T: A \rightarrow B$ a continuous isomorphism. Then $T\left(\partial_{A} S_{A}\right)=\partial_{B} S_{B}$.

Proof: If $x \in \partial_{A} S_{A}$, then there exist sequences $\left(x_{n}\right)$ in $S_{A}$ and $\left(y_{n}\right)$ in $A \backslash S_{A}$ such that $x_{n} \rightarrow x$ and $y_{n} \rightarrow x$. It follows from Lemma 2.13 (3) that $T y_{n} \in B \backslash S_{B}$ and $T x_{n} \in S_{B}$. Since $T$ is continuous, $T x_{n} \rightarrow T x$ and $T y_{n} \rightarrow T x$. Hence $T x \in \partial_{B} S_{B}$.

Conversely, if $y \in \partial_{B} S_{B}$, say $y=T x$ with $x \in A$, then there exist sequences $\left(z_{n}\right)$ in $S_{B}$ and $\left(w_{n}\right)$ in $B \backslash S_{B}$ such that $z_{n} \rightarrow y$ and $w_{n} \rightarrow y$. It follows from Lemma 2.13 (3) that $z_{n}=T x_{n}$ with $x_{n} \in S_{A}$ and that $w_{n} \in B \backslash T S_{A}$, so that $w_{n}=T u_{n}$ with $u_{n} \in A \backslash S_{A}$. Since $T$ is bijective, linear and bounded, $T^{-1}$ exists and is linear and bounded (by the Bounded Inverse Theorem), which implies that $x_{n} \rightarrow x$ and $u_{n} \rightarrow x$. Since $\left(x_{n}\right)$ is in $S_{A}$ and $\left(u_{n}\right)$ is in $A \backslash S_{A}$, it follows that $x \in \partial_{A} S_{A}$.

In the following result $\operatorname{ker} T$ will denote the kernel of $T$.

THEOREM 2.15. Let $A$ and $B$ be Banach algebras, $T: A \rightarrow B$ a continuous isomorphism and $a \in A$. Then

$$
S_{\partial}(a, A)=S_{\theta}(T a, B)=\bigcup_{b \in \operatorname{ker} T} S_{\partial}(a+b, A) .
$$

Proof: If $\lambda \in S_{\partial}(a, A)$, then $\lambda-a \in \partial_{A} S_{A}$, so that Theorem 2.14 implies that $\lambda-T a=T(\lambda-a) \in \partial_{B} S_{B}$, and so $\lambda \in S_{\partial}(T a, B)$.

If $\lambda \in S_{\theta}(a+b, A)$ for some $b \in \operatorname{ker} T$, then $\lambda-T a=T(\lambda-a-b) \in \partial_{B} S_{B}$, by Theorem 2.14, so that $\lambda \in S_{\partial}(T a, B)$.

We have proved that

$$
S_{\partial}(a, A) \subseteq S_{\partial}(T a, B) \text { and } \bigcup_{b \in \operatorname{ker} T} S_{\partial}(a+b, A) \subseteq S_{\partial}(T a, B) .
$$

If $\lambda \in S_{\theta}(T a, B)$, then $T(\lambda-a)=\lambda-T a \in \partial_{B} S_{B}$, so that Theorem 2.14 implies that $T(\lambda-a) \in T\left(\partial_{A} S_{A}\right)$. The injectivity of $T$ implies that $\lambda-a \in \partial_{A} S_{A}$, so that $\lambda \in S_{\partial}(a, A)$. Since $0 \in \operatorname{ker} T$, we obtain the following inclusions:

$$
S_{\partial}(T a, B) \subseteq S_{\partial}(a, A) \subseteq \bigcup_{b \in \operatorname{ker} T} S_{\partial}(a+b, A)
$$

Hence the results follow. 


\section{Applications in ORDERED Banach algebras}

In this section we investigate certain results in ordered Banach algebras involving the boundary spectrum. From ([9, Section 3]) we recall that an algebra cone $C$ of a complex Banach algebra $A$ with unit 1 is a subset of $A$ containing 1 which is closed under the following operations: addition, positive scalar multiplication, and multiplication. If $A$ has an algebra cone $C$, then $A$, or more specifically $(A, C)$, is called an ordered Banach algebra (OBA). If, in addition, $C \cap-C=\{0\}$, then $C$ is called proper.

An algebra cone $C$ of $A$ induces an ordering " $\leqslant$ " on $A$ in the following way:

$$
a \leqslant b \text { if and only if } b-a \in C
$$

(where $a, b \in A$ ). This ordering is reflexive and transitive. Furthermore, $C$ is proper if and only if the ordering has the additional property of being antisymmetric. Considering the ordering that $C$ induces we find that $C=\{a \in A: a \geqslant 0\}$ and therefore we call the elements of $C$ positive.

An algebra cone $C$ of $A$ is called closed if it is a closed subset of $A$. Furthermore, $C$ is said to be normal if there exists a constant $\alpha>0$ such that it follows from $0 \leqslant a \leqslant b$ in $A$ that $\|a\| \leqslant \alpha\|b\|$. It is well known that if $C$ is normal, then $C$ is proper. If $C$ has the property that if $a \in C$ and $a$ is invertible, then $a^{-1} \in C$, then $C$ is said to be inverse-closed. If $B$ is a Banach algebra such that $1 \in B \subseteq A$, then $C \cap B$ is an algebra cone of $B$, and hence $(B, C \cap B)$ is an OBA.

In $[9,8]$, and later in $[4,5,6,7]$, some spectral theory of positive elements in ordered Banach algebras was developed. In particular, we recall the following results:

THEOREM 3.1. ([9, Theorem 4.1(1)]) Let $(A, C)$ be an OBA with $C$ normal. If $a, b \in A$ such that $0 \leqslant a \leqslant b$, then $r(a) \leqslant r(b)$.

We refer to the above property by saying that the spectral radius in $(A, C)$ is monotone.

Theorem 3.2. ([9, Theorem 5.2]) Let $(A, C)$ be an $O B A$ with $C$ closed and such that the spectral radius in $(A, C)$ is monotone. If $a \in C$, then $r(a) \in \sigma(a)$.

Using the boundary spectrum we obtain the following (slightly stronger) analogues of Theorem 3.2 and ([6, Theorem 3.3]):

Proposition 3.3. Let $(A, C)$ be an $O B A$ with $C$ closed and such that the spectral radius in $(A, C)$ is monotone. If $a \in C$, then $r(a) \in S_{\theta}(a)$.

Proof: If $a \in C$, then by Theorem $3.2 r(a) \in \sigma(a)$. Hence $r(a) \in \partial \sigma(a)$ and so $r(a) \in S_{\partial}(a)$.

Proposition 3.4. Let $(A, C)$ be an $O B A$ with $C$ closed and inverse-closed, and such that the spectral radius in $(A, C)$ is monotone. If $a$ is an invertible element of $C$, then $\delta(a) \in S_{\partial}(a)$. 
Proof: If $a \in C$ and $a$ is invertible, then $a^{-1} \in C$, since $C$ is inverse-closed. Proposition 3.3 implies that $r\left(a^{-1}\right) \in S_{\partial}\left(a^{-1}\right)$. Hence $r\left(a^{-1}\right)=1 / \lambda_{0}$ for some $\lambda_{0} \in S_{\partial}(a)$, by Proposition 2.7. Since $r\left(a^{-1}\right)=1 /(\delta(a))$, the result follows.

In the following result $B$ is a subalgebra of $A$ but not necessarily closed in $A$.

THEOREM 3.5. Let $(A, C)$ be an $O B A$ and $B$ a Banach algebra with $1 \in B \subseteq A$.

1. Suppose that the spectral radius in $(A, C)$ is monotone. If $0 \leqslant a \leqslant b$ with $a, b \in B$ and either $\partial \sigma(a, B)=\partial \sigma(a, A)$ or $S_{\partial}(a, B)=S_{\partial}(a, A)$, then $r(a, B) \leqslant r(b, B)$.

2. Suppose that the spectral radius in $(B, C \cap B)$ is monotone. If $0 \leqslant a \leqslant b$ with $a, b \in B$ and either $\partial \sigma(b, B)=\partial \sigma(b, A)$ or $S_{\theta}(b, B)=S_{\partial}(b, A)$, then $r(a, A) \leqslant r(b, A)$.

Proof:

1. Since $B$ is a subalgebra of $A$, we have that $\sigma(b, A) \subseteq \sigma(b, B)$, so that $r(b, A) \leqslant r(b, B)$. The monotonicity of the spectral radius in $(A, C)$ implies that $r(a, A) \leqslant r(b, A)$. Finally, the assumption that either $\partial \sigma(a, B)$ $=\partial \sigma(a, A)$ or $S_{\theta}(a, B)=S_{\theta}(a, A)$ yields $r(a, B)=r(a, A)$, by Proposition 2.1. Combining the results, it follows that $r(a, B) \leqslant r(b, B)$.

2. Similarly as in (1), the fact that $B$ is a subalgebra of $A$, the monotonicity of the spectral radius in $(B, C \cap B)$ and the additional assumption imply, respectively, that $r(a, A) \leqslant r(a, B), r(a, B) \leqslant r(b, B)$ and $r(b, B)=r(b, A)$, which yield the result.

We note that Theorem 3.5 (2) is a stronger version of ([9, Proposition 4.5]).

For our next result we need the following lemma and theorem:

Lemma 3.6. ([7, Lemma 4.1]) Let $A$ be a Banach algebra, $x, y \in A$ and $\alpha \in \mathbb{C}$. If $\alpha-x$ is invertible and $r\left((\alpha-x)^{-1}(x-y)\right)<1$, then $\alpha-y$ is invertible.

TheOREM 3.7. ([7, Proof of Theorem 4.2]) Let $(A, C)$ be an OBA with $C$ closed and normal, and let $x \in C$. If $y \in C$ such that $x \leqslant y$ and either $x y \leqslant y x$ or $y x \leqslant x y$, and $\alpha$ is a positive real number such that $\alpha>r(x)$, then

$$
r\left((\alpha-x)^{-1}(y-x)\right) \leqslant r\left((\alpha-x)^{-1}\right) r(y-x) .
$$

Now let $(A, C)$ be an OBA. Define, for each $x \in C$, an analogue $A^{\prime}(x)$ of the set $A(x)$ (defined in ([7, Section 4])) as follows:

$A^{\prime}(x)=\{y \in A: x \leqslant y, \quad x y \leqslant y x$ or $y x \leqslant x y \quad$ and

$$
\left.d\left(r(y), S_{\partial}(x)\right) \geqslant d\left(\alpha, S_{\theta}(x)\right) \text { for all } \alpha \in S_{\partial}(y)\right\}
$$

Then $x \in A^{\prime}(x), A^{\prime}(x) \subseteq C$ and $A^{\prime}(0)=C$. Finally, the following theorem is a complementary result to $([\mathbf{7}$, Theorem 4.2$])$ : 
Theorem 3.8. Let $(A, C)$ be an $O B A$ with $C$ closed and normal, and let $x \in C$. Then $S_{\theta}(y) \subseteq S_{\theta}(x)+r(x-y)$ for all $y \in A^{\prime}(x)$.

Proof: Let $y \in A^{\prime}(x)$. Then $0 \leqslant x \leqslant y$, so that $r(x) \leqslant r(y)$, by Theorem 3.1. If $r(x)=r(y)$, then $d\left(r(y), S_{\theta}(x)\right)=0$, by Proposition 3.3, so that, by the assumption, $d\left(\alpha, S_{\partial}(x)\right)=0$ for all $\alpha \in S_{\theta}(y)$. This implies that $d\left(\alpha, S_{\theta}(x)\right) \leqslant r(x-y)$ for all $\alpha \in S_{\partial}(y)$, so that $S_{\partial}(y) \subseteq S_{\partial}(x)+r(x-y)$.

So suppose that $r(x)<r(y)$, and suppose there exists an $\alpha \in S_{\theta}(y)$ such that $d\left(\alpha, S_{\partial}(x)\right)>r(x-y)$. Proposition 3.3 implies that $r(y) \in S_{\theta}(y)$ and hence, by the assumption, we may take $\alpha \in \mathbb{R}^{+}$with $\alpha>r(x)$. Since $\alpha \notin \sigma(x)$, it follows from Proposition 2.1 that $d\left(\alpha, S_{\theta}(x)\right)=d(\alpha, \sigma(x))$, so that $d\left(\alpha, S_{\partial}(x)\right)=1 /\left(r\left((\alpha-x)^{-1}\right)\right)$. Therefore $r\left((\alpha-x)^{-1}\right) r(x-y)<1$ with $\alpha \in \mathbb{R}^{+}$and $\alpha>r(x)$.

It follows from Theorem 3.7 that $r\left((\alpha-x)^{-1}(y-x)\right)<1$, so that $\alpha \notin \sigma(y)$, by Lemma 3.6. Hence $\alpha \notin S_{\partial}(y)-$ a contradiction. Therefore $d\left(\alpha, S_{\partial}(x)\right) \leqslant r(x-y)$ for all $\alpha \in S_{\theta}(y)$, so that $S_{\theta}(y) \subseteq S_{\theta}(x)+r(x-y)$.

\section{REFERENCES}

[1] B. Aupetit, A primer on spectral theory (Springer-Verlag, New York, 1991).

[2] F.F. Bonsall and J. Duncan, Complete normed algebras (Springer-Verlag, New York, 1973).

[3] P.R. Halmos, A Hilbert space problem book (Springer-Verlag, New York, 1982).

[4] H. du T. Mouton and S. Mouton, 'Domination properties in ordered Banach algebras', Studia Math. 149 (2002), 63-73.

[5] S. Mouton, 'Convergence properties of positive elements in Banach algebras', Math. Proc. R. Ir. Acad. 102A (2002), 149-162.

[6] S. Mouton, 'A spectral problem in ordered Banach algebras', Bull. Austral. Math. Soc. 67 (2003), 131-144.

[7] S. Mouton, 'On spectral continuity of positive elements', Studia Math. 174 (2006), 75-84.

[8] S. Mouton (née Rode) and H. Raubenheimer, 'More spectral theory in ordered Banach algebras', Positivity 1 (1997), 305-317.

[9] H. Raubenheimer and S. Rode, 'Cones in Banach algebras', Indag. Math. (N.S.) 7 (1996), 489-502.

[10] A.E. Taylor and D.C. Lay, Introduction to functional analysis (Krieger, Florida, 1986).

Department of Mathematical Sciences

University of Stellenbosch

Private Bag X1

Matieland 7602

South Africa

e-mail: smo@sun.ac.za 